\title{
Jacques de Sève illustrateur du théâtre de Jean Racine
} Marie-Claire Planche-Touron

\section{Citer ce document / Cite this document :}

Planche-Touron Marie-Claire. Jacques de Sève illustrateur du théâtre de Jean Racine. In: Dix-huitième Siècle, $n^{\circ} 37,2005$.

Politiques et cultures des Lumières. pp. 513-535;

doi : https://doi.org/10.3406/dhs.2005.2691

https://www.persee.fr/doc/dhs_0070-6760_2005_num_37_1_2691

Fichier pdf généré le 17/05/2018 


\section{JACQUES DE SÈVE ILLUSTRATEUR DU THÉÂTRE DE JEAN RACINE}

Le dessinateur Jacques de Sève a illustré entre 1742 et 1788 de nombreux ouvrages. Malgré une présence remarquable dans le livre illustré de cette période, nous ne connaissons que peu de choses de cet artiste dont les compositions furent gravées par les plus grands noms que compte le $18^{\mathrm{C}}$ siècle (Eisen, Flippart, Le Mirc...). Les dictionnaires anciens ou plus récents ne mentionnent aucune date biographique, nous savons seulement qu'il eut un fils, Jacques-Eustache, lui aussi dessinateur. J. de Sève, dessinateur prolixe, sut orner des ouvrages littéraires, scientifiques ou encore juridiques. À ce jour nous avons recensé une quarantaine de contributions dont nous donnons la liste à la suite de cet article ${ }^{\text {. }}$ Des premières compositions quelque peu maladroites aux œuvres beaucoup plus abouties, l'évolution de son trait est tout à fait perceptible. Les dessins de J. de Sève sont très variés, avec des figures agréables, vivantes et un recours au langage allégorique assez fréquent. Son sens de l'ornement ne manque parfois pas de légèreté ou d'ironie. Les putti remplacent ici et là hommes ou femmes dans des compositions qui en deviennent attachantes. Nous pensons aux bandeaux de l'Histoire naturelle de Buffon : les putti, en compagnie d'animaux ou dans un cabinet de sciences naturelles semblent éloignés du langage allégorique, ils s'approchent davantage du monde de l'enfance ${ }^{2}$. Cet ouvrage mêle l'ornement et le scientifique puisque J. de Sève a exécuté d'une part des bandeaux décoratifs et d'autre part, avec Buvée l'Américain, les planches scientifiques. L'illustration de ces volumes résume à elle seule ce que fut le livre à figures au $18^{\mathfrak{e}}$ siècle.

1. Comme point de départ nous avons tout d'abord utilisé les deux ouvrages de référence sur l'illustration au $18^{\mathrm{c}}$ siècle. H. Cohen, Guide de l'amateur de livres à vignettes. Paris, 1880. R. Portalis, Les dessinateurs d'illustrations all dix-huitième siècle. Paris, 1877. Les titres mentionnés ont été vérifiés et la liste des contributions de J. de Sève s'est étoffée au gré des consultations de catalogues de bibliothèques.

2. Buffon, Histoire Naturelle générale et particulière. Paris. Imprimerie Royale, 1749-1767. $15 \mathrm{vol}$. in-4. 
Le livre illustré reçoit au $18^{\mathrm{c}}$ siècle une iconographie abondante; frontispices et vignettes ne suffisent plus au plaisir des yeux, on orne les pages de bandeaux, de culs-de-lampe qui peuvent être purement décoratifs ou en lien avec le texte ${ }^{3}$. Les œuvres des auteurs du $17^{\mathrm{e}}$ siècle bénéficient de somptueuses éditions illustrées par les plus grands artistes: Molière illustré par Boucher, La Fontaine par Cochin et Oudry ou Corneille par Gravelot ${ }^{4}$. Le goût et la fantaisie rocailles s'expriment aussi dans les livres qui sont de véritables objets de collection. Jacques de Sève a illustré Racine à deux reprises. Ces éditions - surtout celle de 1760 - sont uniques dans l'ensemble des éditions illustrées du poète; elles se démarquent de toutes celles publiées depuis le $17^{\mathrm{e}}$ siècle. Au cours du $18^{\mathrm{c}}$ siècle, on compte en tout sept éditions illustrées de Racine, parues avec une certaine régularité de 1713 à $1796^{5}$. Les premiers dessins de Jacques de Sève sont d'abord destinés à une édition en trois volumes in-octavo publiée chez

3. Sur ces questions, voir C. Michel, C.N. Cochin et le livre illustré au $18^{\prime \prime}$ siècle. Genève, 1987.1751-1770 est la période la plus favorable au décor du livre : «Jamais les livres illustrés n'ont été aussi nombreux et aussi copieusement ornés que pendant cette période. ", p. 80. Voir aussi J. et E. Goncourt, L'art du $18^{\mathrm{e}}$ siècle. Paris, 1928: "Aussi le règne de Louis XV est-il le triomphe de ce qu'on appellera plus tard l’illustration. L’image remplit le livre, déborde dans la page. l'encadre, fait sa tête et sa fin, dévore partout le blanc : ce ne sont que frontispices, fleurons, lettres grises, culs-de-lampe, cartouches, attributs, bordures symboliques », vol. II, p. 235.

4. Molière, CEuvres, Paris, Prault. 1734-1735, 6 vol. in-4. Illustrations gravées d'après François Boucher. Cette édition comporte des bandeaux allégoriques.

J. de La Fontaine, Contes et nouvelles en vers, Paris, David Jeune, 1743, 2 vol., in-8. Vignettes de C.N. Cochin. J de La Fontaine, Fables, Paris, Desaint et Saillant, 1755-1759, 4 vol. in fol. Vignettes d'après J.B. Oudry. Pour les éditions illustrées du poète, voir Claire Lesage (dir.), Jean de La Fontaine. Paris, 1995. Exposition Paris, BnF, 1995.

P. Corneille, Euvres, S.1., 1764. 12 vol. in-8. Vignettes d'après Hubert-François Gravelot.

5. J. Racine, Euvres, Amsterdam, H. Schelte, 1713, 2 vol. in-18. Vignettes de Jacobus Harrewyn.

J. Racine, Fuvres, Londres, J. Tonson J. Watt, 1723, 2 vol. in-4. Vignettes d'après Louis Chéron.

J. Racine, CEuvres, Amsterdam, J.F. Bernard, 1743, 3 vol. in-12. Vignettes d'après Louis-Fabricius Du Bourg.

J. Racine, Euvres, Paris, vve Gandouin, 1750, 3 vol. in-8. Bandeaux et fleurons d'après Jacques de Sève.

J. Racine, CEuvres, Paris, Le Breton, 1760, 3 vol. in-4. Illustrations d'après Jacques de Sève.

J. Racine, Euvres, Paris, Louis Cellot, 1768, 7 vol. in-8. Vignettes d'après Hubert-François Gravelot. 
la veuve Gandouin en 1750. J. de Sève exécute pour chaque volume un fleuron et un bandeau gravés par D. Sornique mais l'édition ne comporte pas de vignettes. Chacun des fleurons et des bandeaux met en scène des personnages qui sont souvent aux frontières de l'enfance dans des compositions de faible qualité 6 . Certains sujets ont été repris dans l'édition de 1760, mais les dessins n'ont pas été réutilisés.

La « grande » édition de Racine est publiée en 1760 chez Le Breton, en trois in-quarto. Elle rassemble les pièces de théâtre et des Euvres diverses. L'ornement est partout : fleurons, vignettes, bandeaux et culs-de-lampe font référence aux textes de Racine. Pour chaque pièce, une vignette sur la page de gauche, un bandeau et des culs-de-lampe. Le format des volumes autorise de belles vignettes dont l'iconographie est en quelque sorte complétée par les autres illustrations. Les putti y prennent la place des personnages dans des compositions où symbolique et allégorique se mêlent souvent. La représentation des personnages de Racine sous les traits d'enfants ou de putti (on peut hésiter sur la terminologie à utiliser) fait référence au langage allégorique auquel d'autres artistes ont eu recours à la même période. Cependant il est difficile de savoir qui de l'éditeur ou du dessinateur présidait à ces choix et d'en connaître les raisons profondes. Les dictionnaires iconologiques du $18^{\mathrm{e}}$ siècle s'inscrivent dans la tradition de Ripa : définir les allégories tout en en donnant une représentation visuelle ${ }^{7}$. J. de Sève a d'ailleurs dessiné en 1756 le frontispice du Dictionnaire iconologique de Lacombe de Prézel ${ }^{8}$; ses compositions témoignent d'une bonne connaissance des figures allégoriques.

J. Racine, Euvres, Paris, Déterville, 1796, 4 vol. in-8. Vignettes d'après JeanJacques-François Le Barbier.

Voir notre thèse d'histoire de l'art consacrée à l'iconographie racinienne: Passions raciniennes et arts visuels. Pictura loquens, 1668-1815, Université de Bourgogne, 2000.

Nous devons rappeler l'article de N. Guibert qui a commenté les éditions illustrées de Racine: N. Guibert, "L'iconographie de Racine à la bibliothèque de l'Arsenal », Cahiers Raciniens, 1970, $1^{\text {ir }}$ semestre, p. 9-151.

6. C'est une édition assez rare. Tome I : fleuron avec trois putti tenant les attributs de la tragédie, bandeau en tête de La Thébaïle représentant le combat des frères ennemis. Tome II : fleuron avec trois putti symbolisant la mort de Britannicus, bandeau en tête de Britannicus représentant le meurtre de ce dernier. Tome III : fleuron avec trois putti symbolisant le suicide d'Ériphile, bandeau en tête d'Iphigénie représentant le suicide d'Ériphile.

7. J.B. Boudard, Iconologie tirée de divers auteurs, Paris, Tilliard, 1759.

C.N. Cochin, Almanach iconologique, Paris, Lattré, 1774-1781.

8. H. Lacombe De Prézel, Dictionnaire iconologique, Paris, Th. de Hansy, 1756. 
La diversité de l'ornement permet d'illustrer plusieurs scènes ou de représenter sous différentes formes un même sujet. Il en est ainsi par exemple de la mort de Britannicus qui est le sujet de la vignette et du bandeau. Sur la vignette [fig. 1], le dessinateur a mis en scène le récit de Burrhus : Britannicus s'effondre sous le regard de Néron et des convives, tandis que Narcisse tente de dissimuler l'aiguière contenant le poison. La disposition, les attitudes, les regards, tout concourt à la compréhension du sujet ${ }^{9}$. Pour le bandeau [fig. 2], des figures allégoriques accompagnent les putti. Britannicus bascule en arrière, laissant choir la coupe de la réconciliation. Néron, la coupe à la main, contemple son forfait tandis qu'à ses côtés un putto appuyé sur un renard tient un masque ; c'est la Fourberie. Enfin, au-dessus de la scène un putto sur une nue, les yeux bandés, le flambeau de l'amour et un poignard dans les mains, figure l'Aveugle Espérance. Pour cette allégorie, J. de Sève a pris des libertés avec les dictionnaires iconologiques qui ne font pas mention du poignard et du flambeau. La composition est parfaitement compréhensible, mais les allégories proposent un résumé de la pièce, rappelant l'espoir que le banquet avait fait naître. Ce recours au langage allégorique est particulièrement intéressant puisque au-delà du verbe, J. de Sève entend livrer le caractère des personnages. Pour illustrer des pièces dans lesquelles les passions mènent l'action, il est tout à fait juste de s'intéresser aux caractères ${ }^{10}$. Les peintres, qui depuis toujours souhaitaient concurrencer les poètes, ont cherché l'éloquence. Cependant les figures allégoriques sont uniques dans l'ensemble des éditions illustrées de Racine ". Aussi, au début de chaque volume, l'éditeur a-t-il jugé nécessaire de décrire et de justifier l'ensemble de l'iconographie. Le sujet de la vignette (avec la mention de l'acte et de la scène) ainsi que sa composition sont commentés. Les autres ornements sont décrits plus rapidement tandis que le langage allégorique est en partie déchiffré.

Les premières études sur les éditions des œuvres de Racine paraissent à la fin du $19^{\circ}$ siècle; les dessins de J. de Sève sont remarqués :

9. La composition reprend des éléments du bandeau de 1750 dans un traitement tout à fait abouti.

10. Nous n'oublions pas Les Plaideurs dont les putti en robe de justice et les petits chiens sont assez savoureux.

11. Il faut tout de même citer l'édition pirate des Giurres de Racine 1678 dont la vignette de Phèdre est une allégorie. 
Le vrai révolutionnaire et novateur fut avec succès Jacques de Sève. Avec lui nous sortons de la noblesse de convention pour entrer à pleines voiles dans le mouvement dramatique, la vie et le fracas. [...] Certes la fleur délicate des créations de Racine est froissée parfois sous ce crayon énergique, mais la vie du moins circule dans le livre, la pensée du poète s'anime et prend corps à chaque page. Peut-être y a-t-il quelque excès dans la profusion des sujets mythologiques et des allégories. C'est un tort sans doute, en commentant Racine, d'abonder si fort en des fictions dont lui-même n'a usé que sobrement, mais c'est un tort que l'on pardonne volontiers à l'artiste en faveur de tant de vignettes charmantes qui flattent à la fois l'imagination et le regard. ${ }^{12}$

Il semble cependant que dès le début du siècle, d'aucuns aient reconnu l'importance de cet ensemble. En effet, le libraire et collectionneur Antoine-Augustin Renouard possédait non seulement l'édition de 1760 (parmi d'autres éditions de Racine), mais aussi les dessins que J. de Sève avait exécutés et les gravures. Ils sont mentionnés dans son catalogue de 1819 et dans celui de la vente de sa collection en $1854^{93}$. Ce sont les deux seules mentions des dessins originaux et depuis la vente du 13 décembre, nous ignorons quel fut le sort de ces œuvres.

Les vignettes, par leurs compositions, les attitudes des personnages et les décors sont parmi les plus séduisantes illustrations de Racine. Sève évite le «gracieux », il parvient à l'éloquence par le mouvement et les gestes énergiques ${ }^{14}$. Les personnages ont des visages expressifs, des attitudes nobles qui conviennent à leurs caractères. On remarque cependant ici ou là des mouvements de lèvres, des sourires qui traduisent quelque ironie. Certaines attitudes éloignent du tragique: lorsque Porus se présente devant Alexandre $(\mathrm{V}, 3)$, le roi des Indes tient à peine debout.

12. A.J. Pons, Les éditions illustrées de Racine, Paris, 1878, p. 18.

13. L'édition qu'il possédait était l'un des deux tirages sur papier de Hollande. A.A. Renouard, Catalogue de la bibliothèque d'un amateur, Paris, A.A. Renouard, 1819, tome III, p. 65. Catalogue d'une précieuse collection de livres, manuscrits, autographes, dessins et gravures composant la bibliothèque de feu M. AntoineAugustin Renouard, Paris, J. Renouard, 1854. «n"1538. Dessins de de Sève pour cette édition in-4 de Racine, avec les gravures et tous les fleurons ainsi que leurs dessins. [...] Quatre-vingt-sept pièces, tant en grands dessins qu'en fleurons et vignettes et toutes leurs gravures correspondantes. Un seul dessin, celui d'Iphigénie, n'a pu être retrouvé » (p. 155).

14. Hubert-François Gravelot, dans l'édition de 1768 cède davantage à une mise en scène gracieuse et ses compositions ont souvent moins de force, les attitudes paraissent plus affectées. 
C'est soutenu par un soldat aux efforts visibles qu'il peut se montrer droit sur ses jambes avec un air fier. Seules les larmes des femmes derrière Alexandre rappellent le drame. La «vérité historique »n'est pas négligée, le décor des vignettes est antiquisant ou orientalisant, tout comme les costumes des personnages. Les palais ont une décoration soignée : le dressoir de la salle de banquet de Britannicus, les statues des appartements de Titus et Bérénice, les braseros et les lourdes tentures de Bajazet ou d'Esther. Le traitement des sujets est donc abouti, et l'iconographie présente quelques nouveautés. J. de Sève a retenu des épisodes déjà illustrés dans les éditions précédentes et comme ses prédécesseurs, il a aussi représenté des faits connus par le seul récit. Mais les règles des arts visuels ne sont pas celles du théâtre et la mort peut être exposée au spectateur. Malgré tout il propose des scèncs qui n'avaient pas encore été illustrées. Pour Bajazet, il représente non pas l'évanouissement d'Atalide, mais Roxane montrant à Bajazet la lettre écrite à Atalide $(\mathrm{V}, 4)$. Pour Mithridate, le sujet ne se retrouve dans aucune édition : Arbate arrache la coupe de poison des mains de Monime (V, 3) ; c'est une des planches les plus réussies avec un mouvement particulièrement vif. Enfin, pour Esther la célèbre scène de l'évanouissement est le sujet du bandeau, tandis que sur la vignette Aman se jette aux pieds d'Esther (III, 5).

Bien sûr, chaque illustration mériterait une longue analyse à la lumière des vers de Racine, mais pour analyser la richesse et la force de l'iconographie déployée par $\mathbf{J}$. de Sève, il nous a paru plus cohérent et plus aisé de nous limiter à une seule pièce. Puisqu'il fallait faire un choix, c'est Phèdre qui a retenu notre attention. Cette tragédie est ornée d'une vignette, d'un bandeau et de cinq culs-de-lampe. Malgré l'annonce de l'éditeur, J. de Sève n'a pas dessiné un cul-de-lampe pour chaque acte de chacune des pièces. Avant de regarder les illustrations, lisons l'avertissement de l'éditeur ${ }^{15}$ pour Phèdre:

À la préface de Phèdre, un monstre marin et le char d'Hippolyte groupés ensemble. Neptune pour seconder les voeux de Thésée, fait sortir du fond des mers un monstre énorme, dont les chevaux d'Hippolyte

15. «On a cru devoir donner, pour la satisfaction du public, une explication simple de toutes les planches contenues dans chaque volume. Les vignettes représentent allégoriquement le caractère de la pièce où elles sont destinées, et les culs-de-lampe qui sont à la fin de tous les actes sont analogues à l'acte où ils sont placés. " Le terme de vignette désigne ici toujours le bandeau. Les illustrations de cette pièce ont toutes été gravées par Jean-Jacques Flipart. 
furent effrayés. Ce jeune prince pousse droit à ce monstre, lui lance un dard et le terrasse. Acte $\mathrm{V}$, scène 6 . La vignette représente allégoriquement Hippolyte, par le symbole de l'innocence, victime des fureurs de l'amour. Une harpie tenant le flambeau de l'amour allégorie de Phèdre dans la I. Scène de l'acte I. Phèdre animée des fureurs de l'amour, tire l'épée d'Hippolyte pour s'en percer. Acte II, scène 5. Pour l'acte III. Thésée irrité contre son fils, invoque le dieux des mers pour le faire périr. Pour l'acte V. Le monstre marin blessé et expirant.

Phèdre et Esther sont les seules pièces dont la préface est ornée. L'intention décorative de ce cul-de-lampe est très affirmée [fig. 3]. Le cadre rocaille courbe est disposé sur un socle au décor de grecques soutenu par des putti tenant le rôle d'atlantes et entourant un cartouche. Une tête au sommet qui évoque celle du monstre et des guirlandes complètent l'ensemble. Basculant hors de la bordure, le monstre envoyé par Neptune nous présente son dos, il paraît poussé par le char renversé d'Hippolyte. Sa queue recouverte d'écaille décrit une volute semblable à celles de l'encadrement et de la fumée s'échappe de ses naseaux. Dans la vignette [fig. 4], J. de Sève fait référence au récit de Théramène $(\mathrm{V}, 6)$, mais il n'a pas choisi d'exposer le cadavre aux regards, comme plusieurs de ses prédécesseurs ${ }^{16}$; il a préféré représenter la vivacité et la combativité d'Hippolyte. Le jeune homme conduit son char d'une main, de l'autre il est prêt à lutter contre le monstre surgi des flots :

Parmi des flots d'écume, un monstre furieux.

Son front large est armé de cornes menaçantes;

Tout son corps est couvert d'écailles jaunissantes;

Indomptable taureau, dragon impétueux,

Sa croupe se recourbe en replis tortueux ${ }^{17}$

La fougue et l'ardeur d'Hippolyte s'opposent à la frayeur des chevaux et de ses compagnons. Sa vaillance lui permet de se redresser sur ses jambes et, le visage serein, de s'apprêter à frapper «d'une main sûre ${ }^{18}$ ». Le dessinateur a représenté le début du récit, la première lutte, il laisse ainsi au spectateur qui ne connaît pas la pièce la possibilité d'espérer en la victoire du

16. Charles Lebrun, pour l'édition de Phèdre parue en 1677 avait le premier représenté Hippolyte mort, Chéron et Dubourg dans les éditions de 1723 et 1743 l'avaient suivi.

17. V, 6, vers 1515-1521. J. de Sève s'est conformé à la description de Théramène, à l'exception des écailles qu'il réserve à la partie inférieure du corps.

18. V, 6, vers 1529. 
jeune homme. Les vaguelettes de l'angle inférieur droit et les écailles du monstre à la queue serpentine permettent d'évoquer le milieu marin. Au second plan les compagnons prennent la fuite tandis qu'à l'arrière-plan se dresse le temple mentionné par le texte de Racine.

Le fils de Thésée est encore le sujet du bandeau [fig. 5]. L'encadrement rocaille, riche de volutes et de conques apparaît comme un décor qui ne limite pas complètement l'espace de la composition puisque les enfants et le monstre viennent en avant du cadre. Au centre, trône le char en partie brisé tandis qu'à droite Hippolyte (sous la forme d'un putto) renversé tend la main vers un agneau tourné vers lui. C'est le symbole de l'Innocence que les dictionnaires ont définie ainsi. Sur une nue, au-dessus du groupe l'Amour furieux tenant un flambeau et un poignard. Enfin, à gauche de la composition le monstre expirant dont la longue queue décrit une volute. Assis sur son dos, un enfant qui tient un trident symbolise Neptune.

Le cul-de-lampe du premier acte [fig. 6], représente une harpie grimaçante aux ailes déployées, aux seins pendants et dont le corps est comme enroulé autour du flambeau de l'amour ${ }^{19}$. Elle se tient devant le carquois et les flèches de Cupidon et le motif est agrémenté d'éléments purement décoratifs.

Pour la fin du deuxième acte, le cul-de-lampe représente un instant de la pièce qu'aucun illustrateur n'a encore retenu : Phèdre tente de se frapper avec l'arme d'Hippolyte [fig. 7]. J. de Sève l'a illustré de manière symbolique puisque les enfants tiennent le rôle des personnages. Sa composition, par sa vivacité est traitée avec efficacité, même si les putti ne peuvent avoir la même force dramatique. Le geste de la fille de Minos est arrêté par le bras d'Enone tandis qu'au second plan Hippolyte apparaît dans une attitude de résignation tout à fait surprenante. La tête baissée, la main droite ouverte, il ne peut que contempler son fourreau vide. J. de Sève a porté son attention sur les derniers vers de la scène (II, 5) et mis en image le geste que Racine a seulement esquissé. Dans l'édition de 1768, Hubert-François Gravelot a retenu cette scène pour sa vignette, représentant l'instant où Phèdre s'empare de l'épée. Bien qu'il n'ait pas eu recours aux putti, sa composition n'est pas particulièrement dramatique; les costumes et le mobilier mais surtout le geste d'Hippolyte ne

19. La représentation qu'en a faite le dessinateur correspond en tout point à la description de Lacombe de Prézel. 
suscitent guère d'émotion. Les vignettes de cette édition répondent plutôt au goût du gracieux que le $18^{\mathrm{c}}$ siècle appréciait ${ }^{20}$.

Dans un gracieux motif rocaille le cul-de-lampe de l'acte III représente le monstre et Thésée [fig. 8]. Le monstre est allongé au premier plan sur une conque, tenant entre ses pattes le trident de Neptune; il attend son heure. Au-dessus, la houle légère et Thésée qui clame sa colère. Cependant, le roi de Trézène n'est pas très convaincant, les sentiments qui l'animent ne se lisent pas sur son visage. J. de Sève a proposé une composition très décorative, c'est un ornement du livre en lien avec une scène de la pièce.

Nous avons remarqué de quelle manière la vignette montre le courage d'Hippolyte et sa vaillance au combat. Le jeune homme affronte le monstre, « il lui fait dans le flanc une large blessure ${ }^{21}$ » avant de mourir. Le monstre blessé et fumant est ainsi le sujet du dernier cul-de-lampe [fig. 9]. L'animal allongé est ainsi exposé aux regards; bien que mort Hippolyte a su triompher et la bête qui sert de conclusion à J. de Sève n'a plus rien d'impressionnant. L'iconographie de Phèdre se concentre sur l'histoire d'Hippolyte puisque toutes les figures l'évoquent. Il eut été sans doute difficile de mettre en scène les tourments de Phèdre, non que J. de Sève manquât d'habileté, mais en raison des putti qui limitent la représentation des sentiments ${ }^{22}$.

Les illustrations de cette édition expriment les liens ténus entre le texte et l'image, mais aussi l'invention des arts visuels pour transposer les vers de Racine. La richesse des pièces du poète a permis à J. de Sève d'offrir des compositions d'une grande qualité. A-t-il lu les pièces ou a-t-il suivi les recommandations de l'éditeur? Il nous plaît de penser qu'il lisait Racine parce que la finesse des représentations nous y autorise. Malgré la profusion, l'abondance décorative, cette édition se montre assez fidèle à Racine. J. de Sève séduit le lecteur, le charme en atténuant

20. Vignette gravée par Jean-Baptiste Simonet. La composition d'Anne-Louis Girodet gravée par Raphaël-Urbain Massard dans l'édition de 1801 a quant à elle une grande force expressive.

21. V, 6, vers 1530 .

22. En revanche, dans la magistrale édition des frères Didot parue en 1801 , Phèdre est le sujet principal des vignettes. Cette édition qui fut illustrée par les élèves de l'école de David compte une planche par acte, dans des compositions qui furent exécutées comme des tableaux. Le néoclassicisme a alors banni l'excès d'ornement du livre.

J. Racine, Euvres, Paris, Pierre Didot l'aîné, 1801, 3 vol. in-fol. 
le sens du drame qu'il réserve essentiellement aux vignettes. Il a représenté des scènes que les autres dessinateurs n'avaient jamais retenues, contribuant ainsi à donner un nouvel élan à l'illustration des pièces de Racine. En effet, les précédentes éditions reprenaient le plus souvent les motifs de l'édition de 1676 illustrée par François Chauveau. Très présent pendant la deuxième moitié du $18^{\mathrm{c}}$ siècle, J. de Sève est un dessinateur dont les compositions méritent un examen attentif. Ses figures sont reconnaissables entre toutes, il manque cependant les dessins originaux qui permettraient de mieux apprécier ses qualités de dessinateur ${ }^{23}$. Ses illustrations montrent qu'il a su répondre aux goûts de ses contemporains, tout en conservant une liberté d'invention remarquable. Comme Portalis, nous pensons que « son illustration de beaucoup la plus importante est celle qu'il a dessinée pour la belle édition des Euvres de Racine. Les grandes figures y sont traitées dans le goût un peu théâtral des de Troy et des Boucher, mais avec beaucoup d'aisance et tout à fait en peintre. [...] Les vignettes et fleurons sont pleins d'invention et de mouvement ${ }^{24} \gg$. Mais cette édition de 1760 ne doit pas masquer l'ensemble du travail de J. de Sève qui reste encore à étudier.

\section{MARIE-Claire PlanCHE-TOURON Université de Bourgogne}

\footnotetext{
23. Le cabinet des Estampes de la BnF conserve des dessins de planches scientifiques de l'Histoire naturelle de Buffon.

24. Voir supra, p. 620.
} 
JACQUES DE SÈVE ILLUSTRATEUR DE RACINE 523

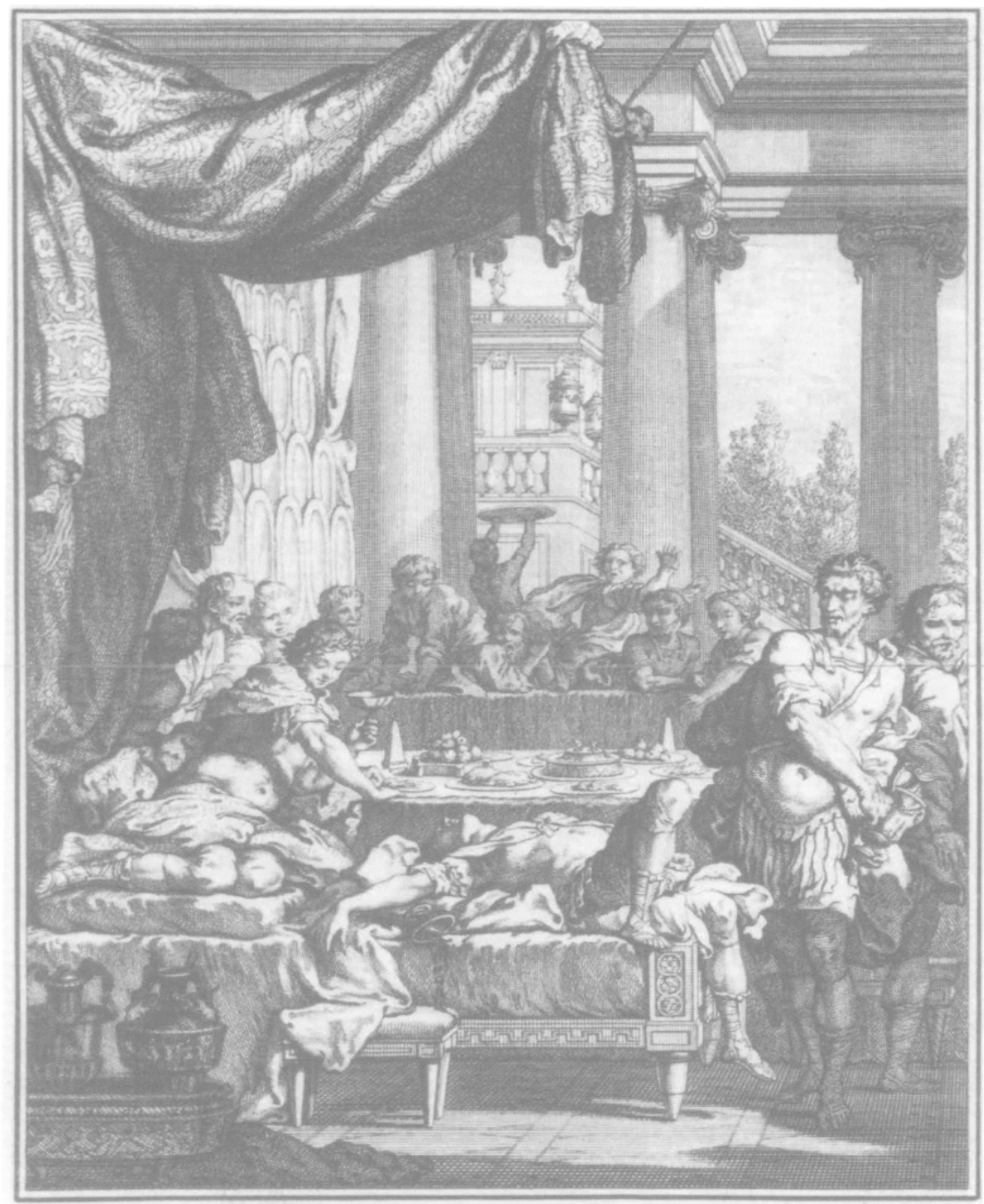

BIRITANNICUS.

Britannicus, vignette, gravure de Dominique Sornique d'après Jacques de Sève. Vol. I, p. 328.

(Clichés de l'auteur avec l'aimable autorisation de la Bibliothèque municipale de Nancy). 


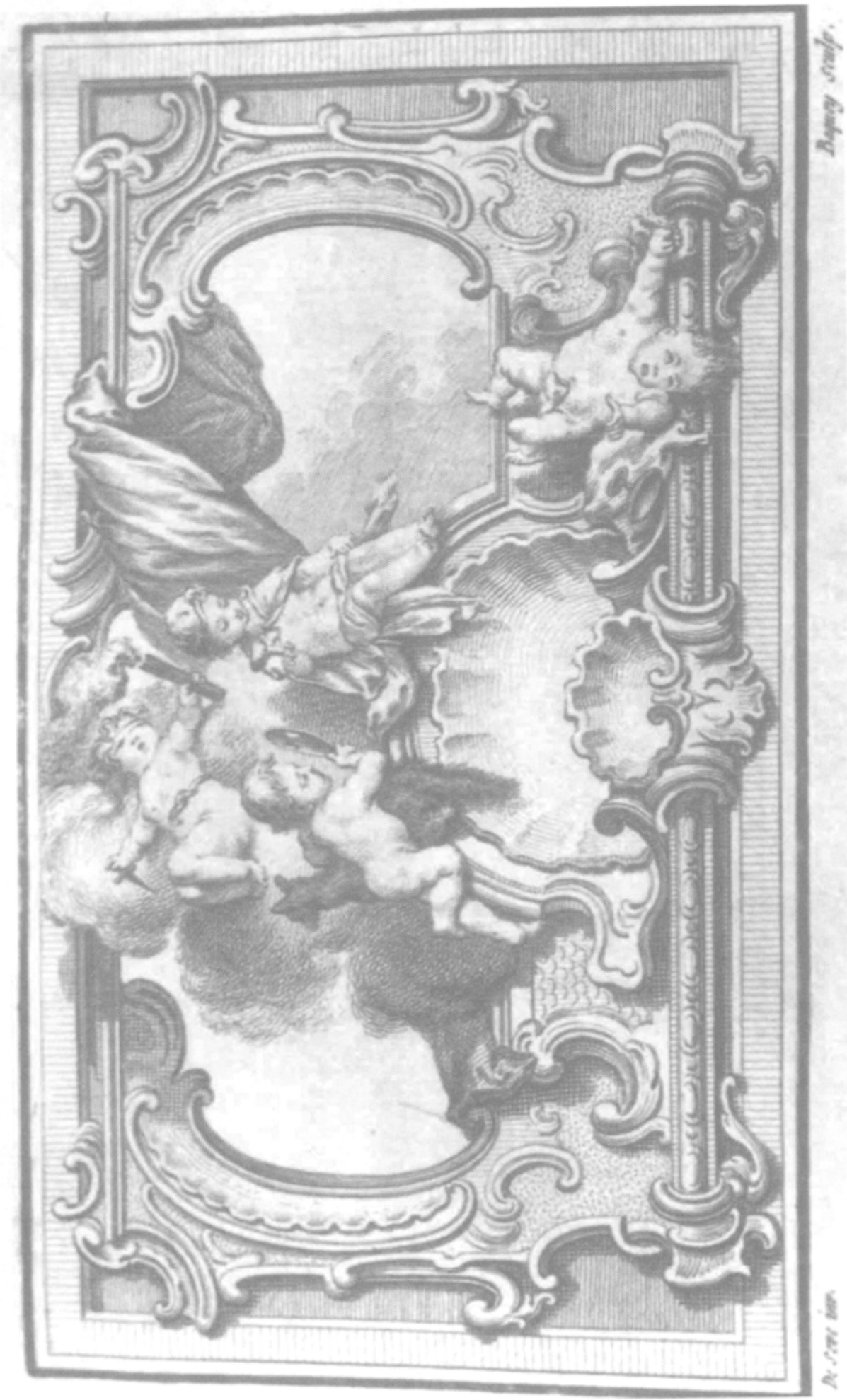

Britannicus, bandeau, gravure de Charles Baquoy d’après Jacques de Sève. Vol. I, p. 329. 
JACQUES DE SÈVE ILLUSTRATEUR DE RACINE 525

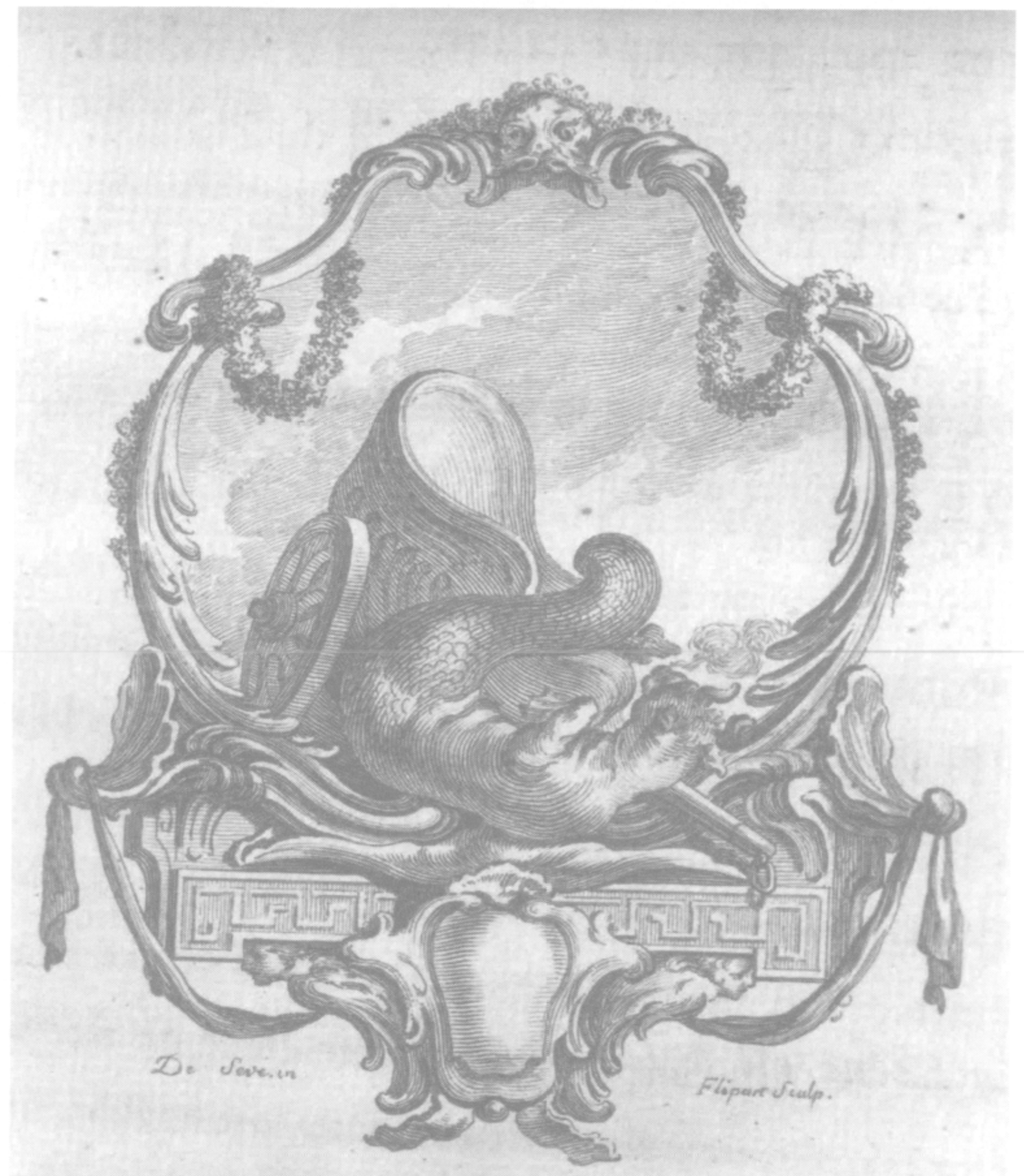

Phèdre, cul-de-lampe préface, gravure de Jean-Jacques Flipart d'après Jacques de Sève. Vol. II, p. 367. 


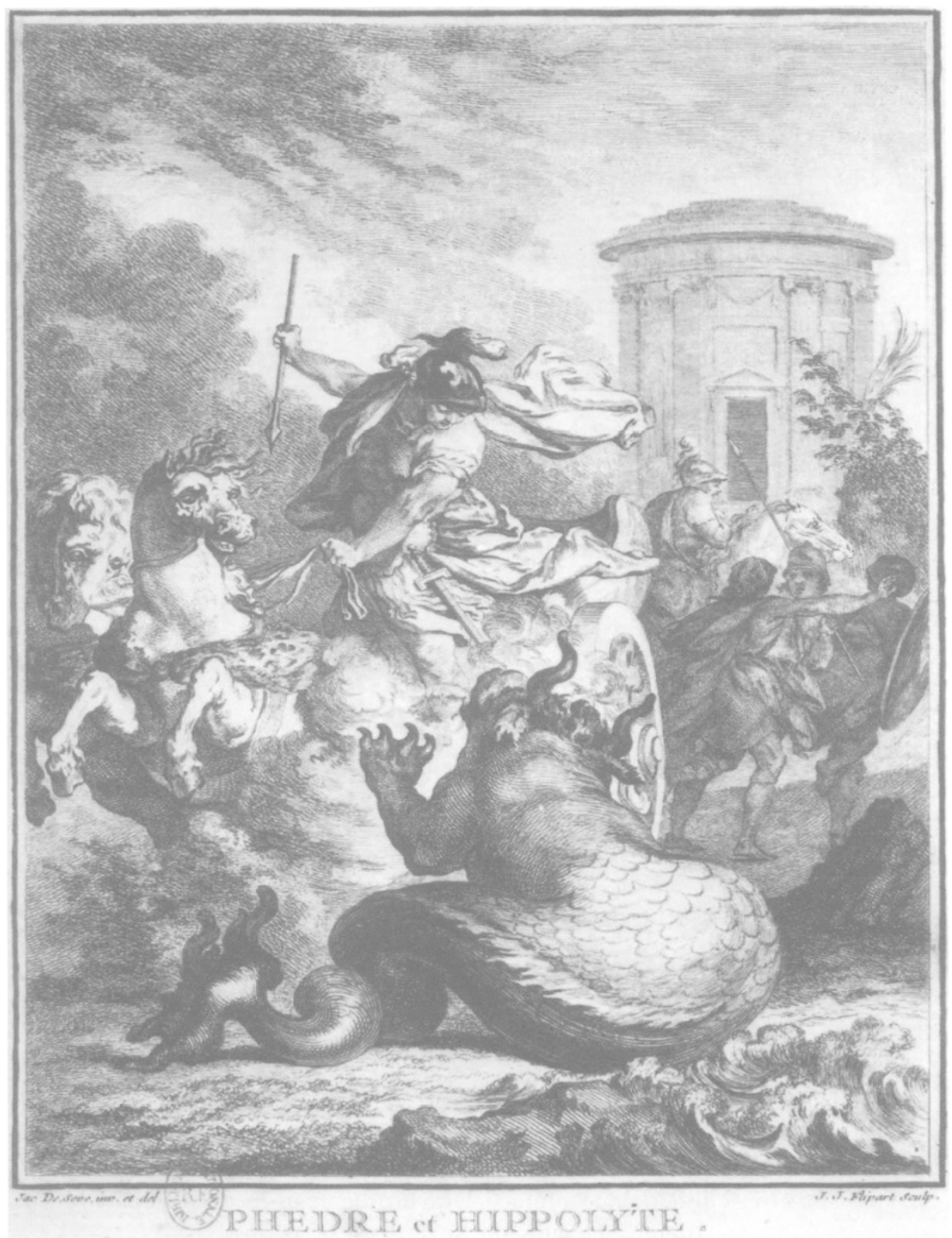

Phèdre, vignette, gravure de Jean-Jacques Flipart d'après Jacques de Sève. Vol. II, p. 368. 


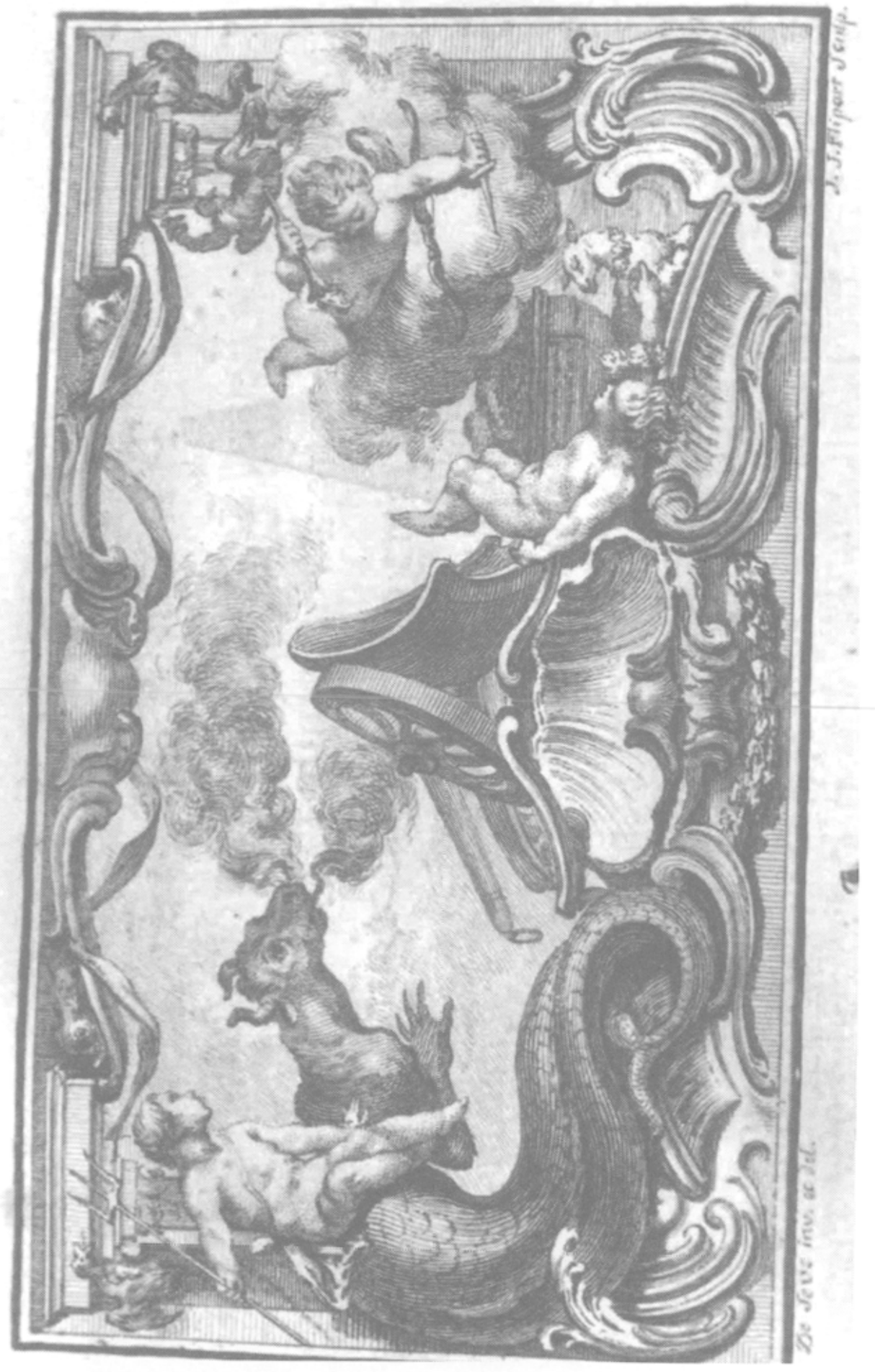

Phèdre, bandeau, gravure de Jean-Jacques Flipart d'après Jacques de Sève. Vol. II, p. 369. 


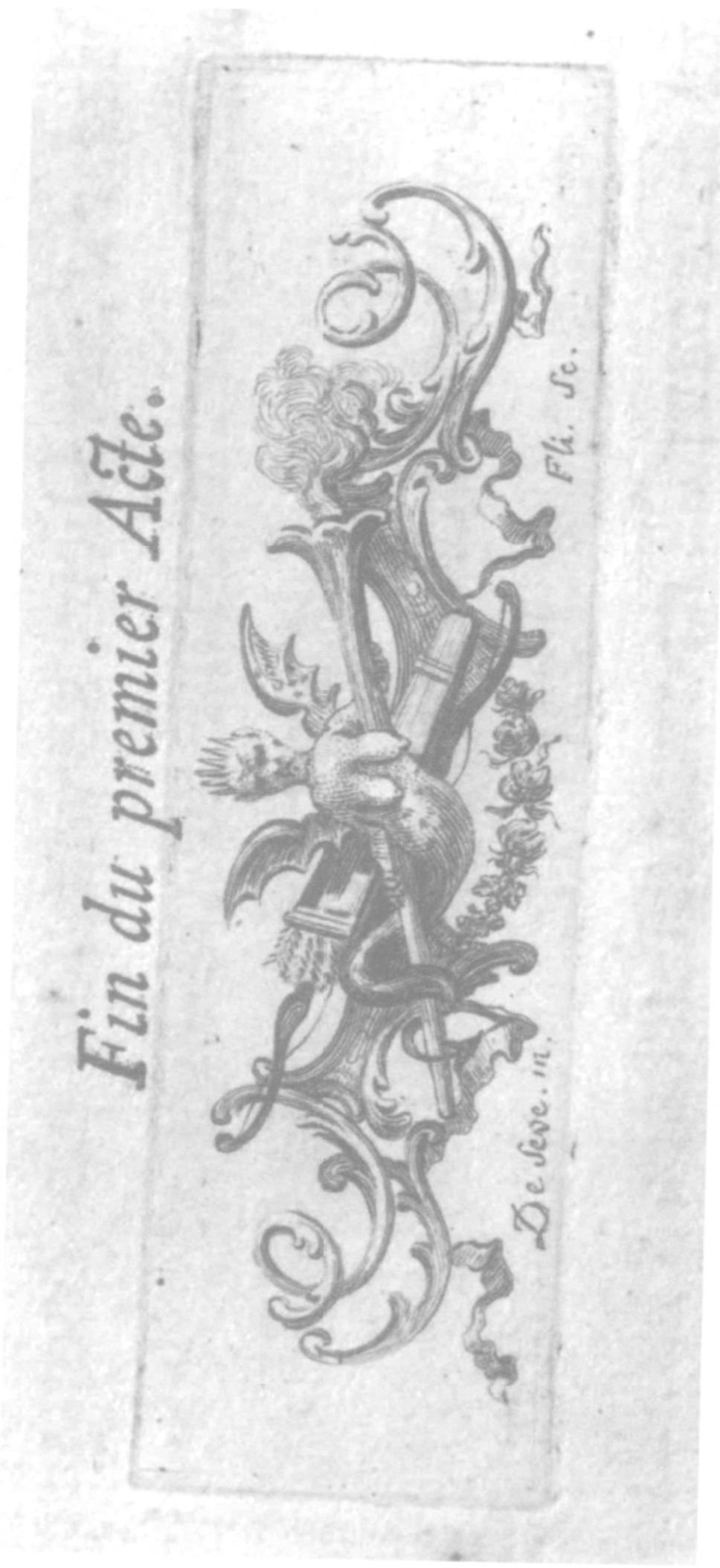

Phèdre, cul-de-lampe acte I, gravure de Jean-Jacques Flipart d'après Jacques de Sève. Vol. II, p. 386. 
JACQUES DE SĖVE ILLUSTRATEUR DE RACINE 529

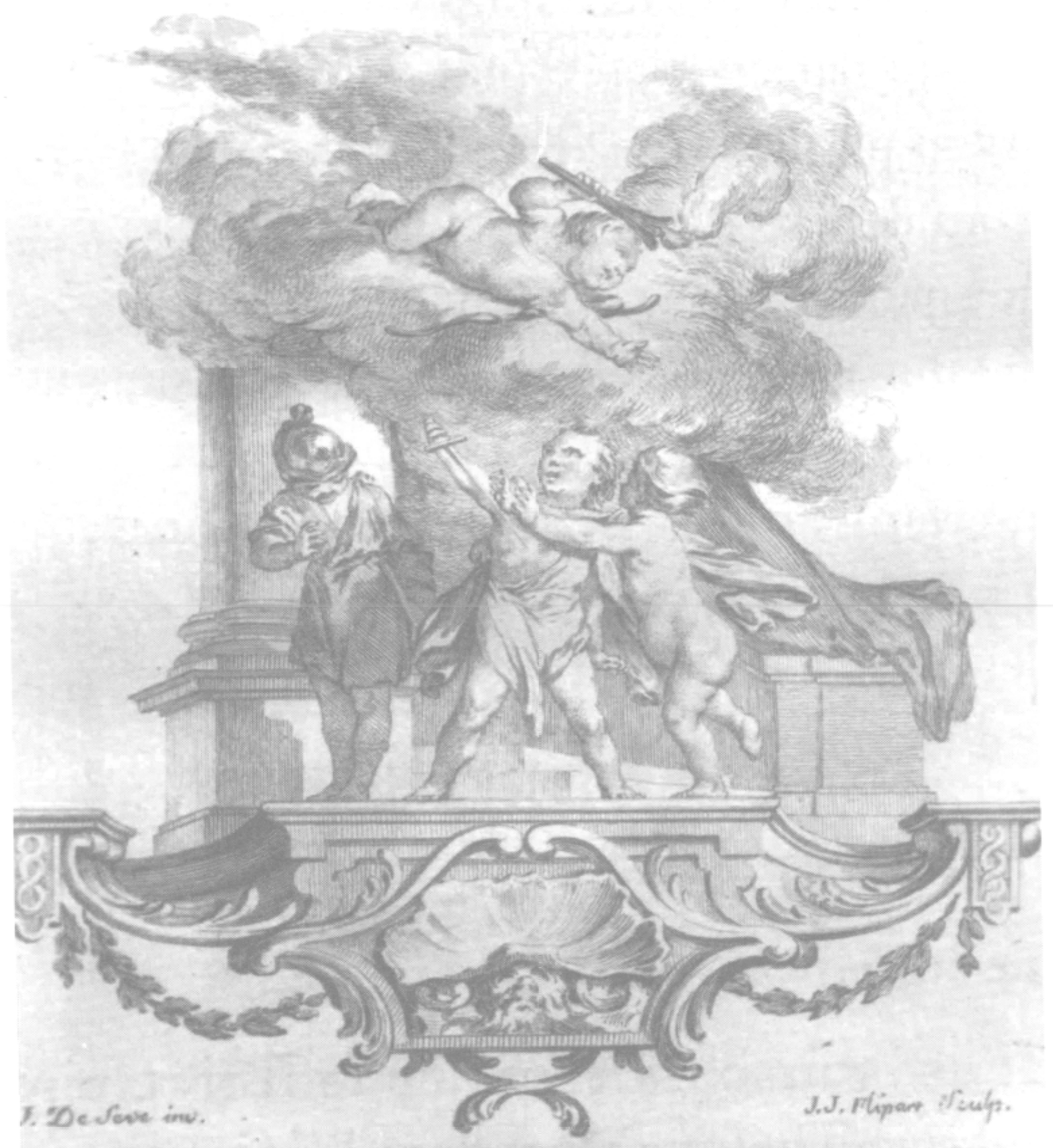

Phèdre, cul-de-lampe acte II, gravure de Jean-Jacques Flipart d'après Jacques de Sève. Vol. II, p. 404. 


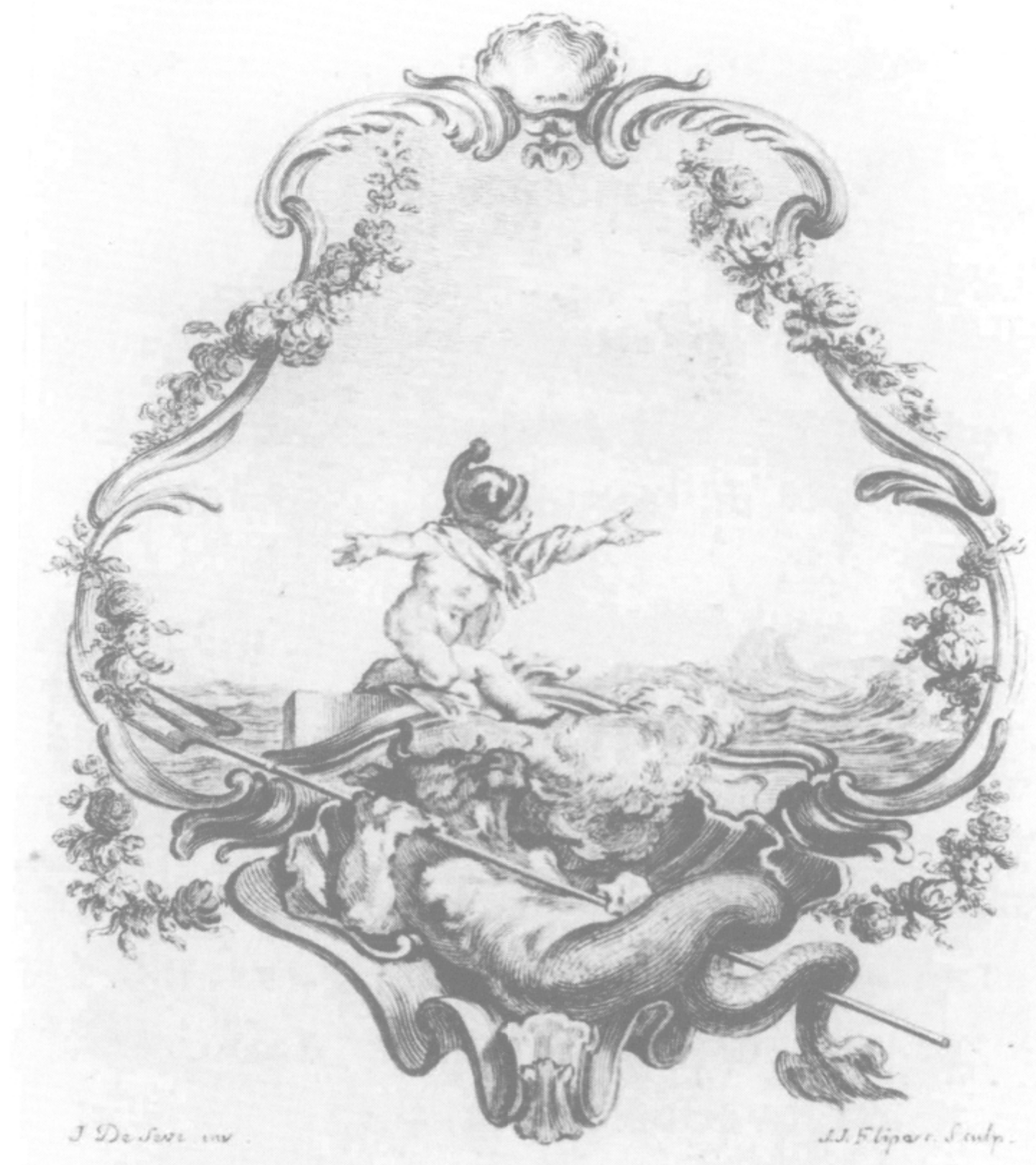

Phèdre, cul-de-lampe acte III, gravure de Jean-Jacques Flipart d'après Jacques de Sève. Vol. II, p. 417. 


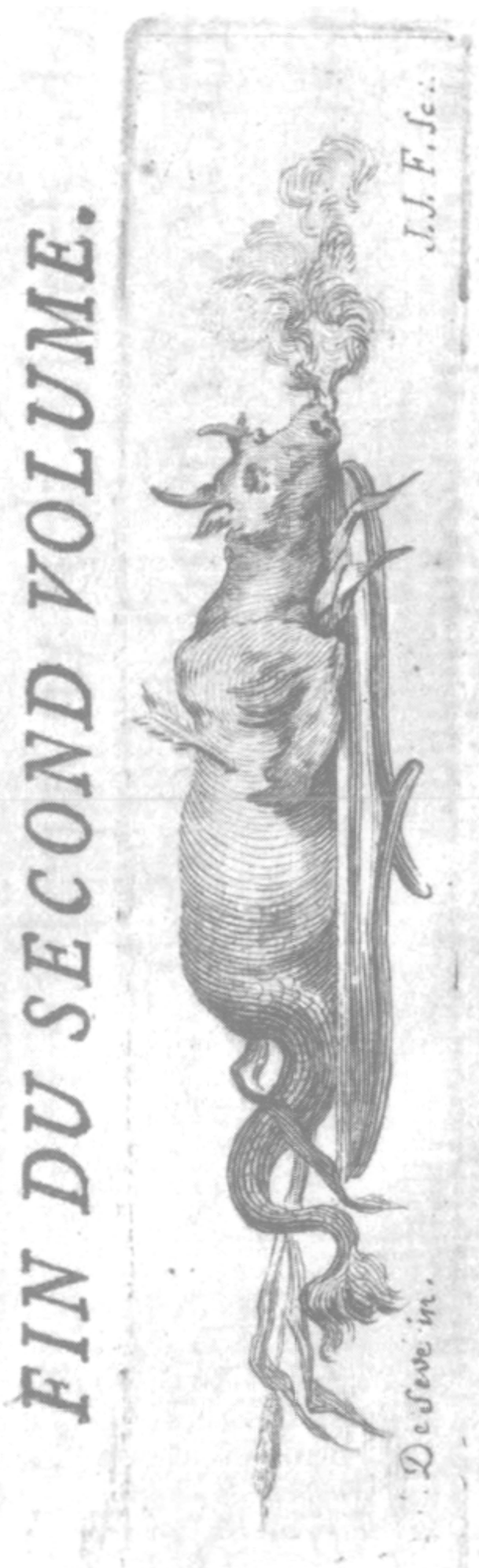

Phèdre, cul-de-lampe acte $\mathrm{V}$, gravure de Jean-Jacques Flipart d'après Jacques de Sève. Vol. II, p. 447. 


\section{Liste des ouvrages illustrés par Jacques de Sève}

1. Allégorie sur le Mariage de Mgrle Dauphin [Louis avec MarieAntoinette d'Autriche, 16 mai 1770]. Vignette J. de Sève gravée par Baquoy.

2. Aguesseau, Henri-François d', Euvres... publiées par l'abbé André, son bibliothécaire, Paris, Libraires Associés, 1759-1789, 13 vol. in-4. Tome V : vignette sur page de titre, bandeaux, culsde-lampe J. de Sève gravés par P. Pate.

3. Argenville, Antoine-Joseph Dézallier d', Abrégé de la vie des plus fameux Peintres avec leurs portraits gravés en taille-douce, les indications de leurs principaux ouvrages, Quelques Réflexions sur leurs caractères et la manière de connaître les desseins des grands Maîtres. Par M.*** de l'Académie Royale des Sciences de Montpellier, Paris, De Bure l'Aîné, 1745. Tome II : handeau avis du libraire J. de Sève gravé par E. Fessard.

4. BatteuX, Abbé Charles, Cours de Belles-Lettres ou Principes de la littérature, Paris, Desaint et Saillant, 1753, 4 vol. in-12. Frontispice, cinq vignettes, quatre fleurons J. de Sève gravés par Ch. Eisen, Delafosse et Baquoy.

5. BUfFON, Georges-Louis Leclerc comte de, Histoire Naturelle générale et particulière, Paris, Imprimerie Royale, 1749-1767. 15 vol. in-4. Bandeaux et planches J. de Sève gravés par F.A. Aveline, P.E Babel, P.F. Basan, Baquoy, A.J. de Fehrt, E. Fessard, L. Legrand, P. Martinasi, J.G. Moitte, D. Sornique, P.F. Tardieu.

6. Carrières, Louis de, Sainte Bible cont. l'ancien et le nouveau testament avec un commentaire littérale inséré dans la trad. Française par le R.P. de Carrières... Paris, Huart et Moreau, Desaint, 1750, 6 vol. in-4. Frontispice, six vignettes J. de Sève gravés par J.J. Pasquier.

7. ClÉment, François - BRIAL, Michel-Jean-Joseph, Recueil des historiens des Gaules et de la France, Paris, veuve Desaint, 17701781, 24 vol. in-fol. Tomes X à XIII : vignettes J. de Sève gravées par C. Baquoy, F. Godefroy, J.M. Moreau et C.N. Malapeau.

8. DeSHOUlières, $\mathbf{M}^{\mathrm{me}}$, CEuvres de Mme et Mlle Deshoulières, nouvelle édition, Paris, Durand, 1753, 2 vol. in-12. Deux bandeaux J. de Sève gravés par J. Ouvrier et P.F. Tardieu.

9. Duhamel du Monceau, Henri-Louis, Traité des arbres fruitiers, contenant leur figure, leur description, leur culture... Paris, Saillant Desaint, 1768, 2 vol. in-4. Frontispice J. de Sève gravé par N. Delaunay.

10. Fournier, Épreuves de deux petits caractères nouvellement gravés et exécutés dans toutes les parties typographiques, par Fournier le jeune, graveur et fondeur de caractères, Paris, 1767, in-32. Frontispice J. de Sève gravé par E. Fessard.

11. FOURniER, Manuel typographique, utile aux gens de lettres, \& à ceux qui exercent les différentes parties de l'Art de l'imprimerie, 
par Fournier le Jeune, Paris, Barbou, 1763, 2 vol., in-8. Tome II : frontispice J. de Sève gravé par E. Fessard.

12. Frascator, Jérôme, Syphilis ou le mal vénérien, Paris, JacquesFrançois Quillau, 1753, in-4. Vignette au titre J. de Sève gravée par Baquoy.

13. GAUTIER DE SiberT. Histoire des Ordres royaux, hospitaliers et militaires de Notre-Dame de Mont-Carmel et de Saint-Lazare de Jérusalem, Paris, Imprimerie Royale, 1772, in-4. Vignettes, culsde-lampe J. de Sève.

14. Hamilton, Antoine, CEuvres mêlées en prose et en vers, Delespine, Charles-Jean-Baptiste, 1749, in-12. Tomes III à VI : vignette au titre, bandeau J. de Sève, gravés par D. Sornique.

15. Hénault, le président, Pièces de Théâtre, en vers et en prose (par le président Hénault), 1770, in-8. Réveil d'Epiménide, bandeau J. de Sève gravé par $\mathrm{Cl}$. Duflos le jeune.

16. LA BRUYèkE, Les Caractères de Thëophraste, avec les Caractères ou les mours de ce siècle, par $M$. de La Bruyère. Nouvelle édition augmentée de quelques notes par M. Coste, Paris, M. E. David père, 1750, 2 vol. in-12. Tomes I et II : frontispices et titres J. de Sève gravés par E. Fessard.

17. La Fontaine, Jean de, Fables choisies, mises en vers par $M$. de la Fontaine, s.l, 1746, 2 vol. in-12. Tome I : bandeau et fleuron de titre J. de Sève gravés par E. Fessard.

18. LA FONTAINE, Jean de, Fables choisies, mises en vers par M. de la Fontaine, avec un nouveau commentaire par M. Coste, nouvelle édition ornée de figures en taille-douce, Paris, M.E. David, 1746, 2 vol. in-12. Frontispice, vignettes J. de Sève gravés par E. Fessard.

19. LA PORTE, Joseph de - LA CROIX, Jean-François de, Histoire littéraire des Femmes françoises, ou Lettres historiques et critiques, contenant un précis de la vie et une analyse raisonnée des ouvrages des femmes qui se sont distinguées dans la littérature françoise, par une société de gens de lettres, Paris, chez Lacombe, 1769, 5 vol., in-8. Frontispice J. de Sève gravé par B.L. Prévost.

20. La Vallière, Duc de, Bibliothèque du Théâtre-François depuis son origine, contenant un extrait de tous les ouvrages composés pour ce théâtre depuis les mystères jusqu'aux pièces de Corneille... Dresde, Michel Groll (Paris, Bauche), 1768, 3 vol. in-8. Tome I et III : culs-de-lampe J. de Sève gravés par E. Fessard.

21. La Vallière, Duc de, Les infortunés amours de Cominge, romance, Paris, N. Delormel, 1752, in-8. Fleuron J. de Sève gravé par E. Fessard.

22. LACOMBE, Jacques, Dictionnaire portatif des beaux-arts ou abrégé de ce qui concerne l'architecture, la sculpture, la peinture, la gravure, la poésie et la musique, Paris, veuve Estienne, J.T. Herissant, 1752. in-8. Bandeau J. de Sève gravé par J.P. Le Bas.

23. LACOMBe de PRézel, Honoré, Dictionnaire iconologique, Paris, Théodore de Hansy, 1756, in-4. Frontispice J. de Sève gravé par P. Baquoy. 
24. LENGLET DU FRESNOY, Nicolas (ed.), Recueil de romans historiques, Londres, sn, 1747. 8 vol. in-8. Fleurons J. de Sève gravés par E. Fessard.

25. Lévesque de Pouilty, Louis-Jean, Théorie des Sentiments agréables, Paris, David le jeune, 1749, in-8. Frontispice, fleuron et trois vignettes J. de Sève gravés par E. Fessard.

26. Maclaurin, Colin, Exposition des Doctrines philosophiques de M. le chevalier Newton. Traduit de l'anglais par M. Laviotte, Paris, 1749, in-4. Portrait, six vignettes J. de Sève gravés par Moitte.

27. MaCquer, Philippe, Abrégé chronologique de l'histoire ecclésiastique, Paris, J.T. Hérissant, 1751, 2 vol., in-8. Dix-sept bandeaux, un fleuron de titre J. de Sève gravés par F.A Aveline, Baquoy et D. Sornique.

28. Magnières, Pierre-André O'Heguerty, comte de, Remarques sur plusieurs branches de commerce et de navigation, s.l., n.n., 1757, in-8. Frontispice J. de Sève gravé par L. Legrand.

29. Maret, Hugues, Encyclopédie méthodique... Paris, Panckoucke Agasse, Liège, Plomteux, 1786-1815, 6 vol. de texte, 1 vol. de planches in-4. Planches J. de Sève?

30. Moncrif, F.A. Paradis de, QEuves de M. de Moncrif, lecteur de la reine, l'un des quarante de l'Académie françoise... Paris, Brunet, 1761, 3 vol. in-16. Titre frontispice, quatre vignettes J. de Sève gravés par Baquoy, P. Chenu, D. Sornique et J. Tardieu.

31. Montesquieu, Ch. Secondat de, Le Temple de Gnide, revu, corrigé et augmenté, Paris, J.M. Huart, 1742, in-8. Titre gravé avec fleuron, frontispice et sept vignettes attribués à J. de Sève, mais non signés.

32. Montesquieu, Ch. Secondat de, Euvres, Londres, Nourses, 1767 , 3 vol. in-4. Frontispice J. de Sève gravé par Littré.

33. MUSIER, J.B. Guillaume, Étrennes aux dames, avec le calendrier de l'année 1763... contenant une notice des femmes illustres dans les belles-lettres, et une notice des livres composés par des femmes, Paris, Musier, in-12. Vignette J. de Sève gravée par D. Sornique.

34. Perrault, Charles, Histoires ou Contes du Temps passé, avec des moralités, par Charles Perrault. Nouvelle édition, augmentée d'une nouvelle à la fin, La Haye - Paris, Coustelier, 1742, in-12. Un frontispice et huit vignettes en-tête par J. de Sève gravés par S. Fokke.

35. Piccolomini Petra, Augusta Caterina, Conseils d'une mère à son fils, poème traduit de l'italien par le Sieur Pingeron, Paris, Vente, 1769, in-12. Frontispice J. de Sève gravé par F. Aveline.

36. RACINE, Jean, Euvres, Paris, veuve Gandouin, 1750, 3 vol. in-12. Bandeaux et fleurons J. de Sève gravés par D. Sornique.

37. Racine, Jean, Euvres, Paris, Le Breton, 1760, 3 vol. in-4. Vignettes, bandeaux et culs-de-lampe J. de Sève gravés par J. Aliamet, C. Baquoy, Chevillet, J.J. Flipart, Legrand N. Le Mire, L.S. Lempereur, D. Sornique, J. Tardieu. 
38. SAINT-Aubin, Le Désaveu de la Nature, nouvelles lettres en vers, À Londres et Paris, Fetil, 1770, gr. in-8. Frontispice et deux J. de Sève gravés par J. Massard et F.D. Née.

39. Saverien, Alexandre, Histoire des Progrès de l'Esprit humain, dans les sciences naturelles et dans les arts qui en dépendent, .... Paris, Lacombe, 1766-1778, 4 vol. in-8. Frontispice J. de Sève gravée par J. Massard.

40. SCARron, Paul, Roman comique, Paris, David, Durand, Pinot, 1752,3 vol. in-12. Vignette J. de Sève gravée par Baquoy.

41. Valmont de Bomare, Jacques Christophe, Dictionnaire raisonné d'histoire naturelle... Paris. Brunet, 1775,8 vol. in-8. Tome I : frontispice J. de Sève gravé par N. Delaunay.

42. Velleius Paterculus et Florus, Caii Velleii Paterculi Histrice romance libri duo (contenant aussi Florus), Paris, Barbou, 1754, in12. Frontispice, bandeau J. de Sève gravés par E. Fessard. 